\section{PSICOLOGÍA IBEROAMERICANA}

\section{Psicología Iberoamericana}

ISSN: 1405-0943

psicología.iberoamericana@uia.mx

Universidad Iberoamericana, Ciudad de México

México

Ramos, Valentina; Jordão, Filomena

Relación entre el conflicto de valores, el sector y el estrés laboral: un modelo descriptivo

Psicología Iberoamericana, vol. 23, núm. 1, enero-junio, 2015, pp. 16-27

Universidad Iberoamericana, Ciudad de México

Distrito Federal, México

Disponible en: http://www.redalyc.org/articulo.oa?id=133944230003

- Cómo citar el artículo

Número completo

- Más información del artículo

- Página de la revista en redalyc.org

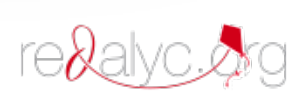

Sistema de Información Científica

Red de Revistas Científicas de América Latina, el Caribe, España y Portugal Proyecto académico sin fines de lucro, desarrollado bajo la iniciativa de acceso abierto 


\title{
Relación entre el conflicto de valores, el sector y el estrés laboral: un modelo descriptivo
}

\author{
Relation Between Value Conflicts, the Sector, and Work-Related Stress: \\ A Descriptive Model
}

\author{
Valentina Ramos \\ Filomena Jordão \\ UnIVERSIDADE DO PORTO
}

\section{RESUMEN}

El estrés laboral, de acuerdo con el modelo transaccional, es un proceso resultante de la relación entre el entorno laboral y los trabajadores. Sin embargo, no se ha considerado la influencia del contexto organizacional, definido por el conflicto entre valores personales y organizacionales y el sector una empresa. El presente estudio tiene por objetivo elaborar un modelo de relaciones entre el conflicto de valores, el sector y proceso de estrés laboral. Para el estudio utilizamos una muestra constituida por 310 trabajadores distribuidos en dos organizaciones de diferente sector y pareados de acuerdo con el género y la edad. Como principal resultado obtuvimos un modelo con buenos índices de ajuste, que describe el efecto mediador de la vivencia de estrés y del conflicto de valores en las relaciones entre las variables. Esto justifica la importancia del contexto en el estudio del estrés laboral.

Palabras clave: estrés laboral, conflicto de valores, sector de la organización, fuentes de estrés, estrategias de coping, modelo de ecuaciones estructurales

\begin{abstract}
Work-related stress, according to the transactional model, is a process born of the relation between the workplace environment and workers. However, no consideration has been given to the influence of the organizational context, defined by the conflict between personal and organizational values and the organization's sector as part of this process. This paper aims to draw up a model of relations between value conflict, the sector and process, and work-related stress. We used a sample of 310 workers distributed in two organizations from different sectors and paired by gender and age. The principle result of our work was the production of a model with good adjustment indexes, which describes the mediating effect of the experience of stress and the conflict of values in the connections between the variables. This justifies the importance of the context in the study of stress in the workplace.
\end{abstract}

Keywords: workplace stress, value conflict, organization sector, sources of stress, coping strategies, structural equations model.

Fecha de recepción: 18 de marzo de 2014 - Fecha de aceptación: 18 de noviembre de 2014

Nota de Autor. Valentina Ramos; Filomena Jordão, Faculdade de Psicologia e de Ciências da Educação, Universidade do Porto.

Este estudio tuvo el apoyo de la Fundación para la Ciencia y la Tecnología (FCT), Referencia: SFRH/ BD/ 43315/ 2008.

La correspondencia relacionada con este artículo deberá estar dirigida a Valentina Ramos, Faculdade de Psicologia e de Ciências da Educação, Universidade do Porto, Rua Alfredo Allen, 4200-135 Porto, Portugal; Teléfono: (+351) 226079 700, e-mail: valia_ramos@yahoo.es 


\section{INTRODUCCIÓN}

El estrés laboral aparece como un fenómeno lleno de múltiples facetas, siendo estudiado desde la perspectiva del trabajador, desde la perspectiva de la organización y desde la interacción entre ambos (Ganster \& Rosen, 2013; Mark \& Smith, 2008). El modelo transaccional de Monat y Lazarus (1991) constituye una de las principales referencias en relación con esta interacción, una vez que consideró la vivencia de estrés laboral como un proceso, introduciendo a los estresores y las respuestas al estrés como sus variables. Sin embargo, perspectivas más recientes sobre el estrés en las organizaciones han considerado también la especificidad de esta vivencia de acuerdo al contexto laboral (Erkutlu, Chafra, \& Bumin, 2011; Ramos \& Jordão, 2012a, 2013a; Singh \& Singh, 2009).

En este sentido, el contexto, definido tanto por la cultura como por otros elementos, resulta relevante en el estudio del estrés laboral. Esto es debido al efecto del conflicto entre valores personales y organizacionales como parte de la estructura de la cultura y del efecto del sector como elementos no culturales de la organización (Morais, Ramos, \& Jordão, 2012; Ramos \& Jordão, 2012a, 2013a). La cultura, además, va a tener un impacto como mediador perceptual de la vivencia de estrés laboral, haciendo que en las organizaciones la vivencia de estrés pueda ser considerada como estrés colectivo (Lansisalmi, Peiró, \& Kivimaki, 2000; Peiró, Zurriaga, \& González-Romá, 2002). Por su parte, teniendo en cuenta al sector, encontramos varios estudios que identificaron diferencias entre el sector público y el privado en relación con la vivencia de estrés, los estresores y las estrategias de afrontamiento (Macklin, Smith, \& Dollard, 2006; Ramos \& Jordão, 2013a, 2013b; Sinha, 2012).

Sin embargo, a pesar de la importancia del contexto en la vivencia de estrés laboral (Latack \& Havlovic, 1992; Nazari \& Emami, 2012; Srivastav, 2009), no hemos identificado estudios que expliquen la relación entre el sector de la organización, el conflicto de valores y el estrés laboral, considerando tanto a los estresores como a las estrategias de coping. Un modelo que describa estas relaciones permitiría a las organizaciones utilizar formas de gestión del estrés laboral de forma más efectiva, a partir de crear la posibilidad de desarrollar estrategias de coping al estrés en función de sus características contextuales, al mismo tiempo que direccionaría el trabajo de identificación y gestión de valores en función de desarrollar mecanismos que eliminen posibles conflictos con los trabajadores y, consecuentemente, disminuir la vivencia de estrés derivada de este conflicto.

\section{El estrés laboral}

Éste ha sido uno de los elementos estudiados en términos de salud de la organización y del trabajador, debido a su impacto tanto a nivel individual como de resultados organizacionales. De acuerdo con Ganster y Rosen (2013), los estudios sobre el estrés en las organizaciones se han centrado en los elementos del contexto laboral que demandan una respuesta de los trabajadores, en la respuesta de los trabajadores a esta demanda $\mathrm{y}$ en el proceso de relaciones que se establece entre ambos. Así, el modelo transaccional de Monat y Lazarus (1991), correspondiente con la consideración del estrés como proceso, ha sido uno de los más utilizados por los investigadores que comparten esta perspectiva.

El estrés laboral aparece entonces como el resultado de la influencia de una serie de elementos considerados como estresores o fuentes de estrés, dando origen a estrategias de afrontamiento o coping que los trabajadores van a utilizar en función de atenuar, disminuir o eliminar el efecto negativo que la vivencia de estrés va a proporcionar (Goh, Sawang, \& Oei, 2010; Mark \& Smith, 2008; Ramos \& Jordão, 2013b). Sin embargo, esta vivencia está condicionada por las características del contexto de la organización.

\section{El contexto en las organizaciones definido por la cultura}

Las características del entorno en el que coexisten las organizaciones con su sistema de relaciones hacen que los estudios que se realicen desde el campo de la psicología organizacional tengan que considerar una amplia gama de factores, donde la cultura de las organizaciones y los trabajadores va a estar constantemente expuesta a la influencia de la otras culturas organizacionales e individuales (Silverthorne, 2005). Los estudios donde se comparan culturas se han denominado como cross-culturales o transculturales. Este tipo de investigación ha tenido un impacto en términos de enfa- 
tizar la importancia del contexto para la explicación de fenómenos comunes a más de un ámbito cultural, pero que tienen origen e implicación diferentes, por lo que no necesariamente pueden ser generalizados.

Dentro de los elementos que componen la cultura, aparecen los valores como aquel que va a permanecer de forma más estable y que, en consecuencia, va a influir en todos los comportamientos organizacionales. Los valores han sido utilizados, por este motivo, como los determinantes del tipo de cultura que predomina en una organización (Dolan \& García, 2002; Hofstede, Neuijen, Ohayv \& Sanders, 1990; Schein, 1996; Schwartz, 1992). La influencia de los valores va a estar orientada hacia la forma en que las personas perciben su entorno, se perciben a sí mismo y perciben a los otros (Silverthorne, 2005).

\section{El conflicto de valores organizacionales y personales, y su relación con el estrés laboral}

Los valores de una empresa están considerados como los elementos que, en última instancia, son los verdaderos determinantes del comportamiento y la estructura organizacional (Franke, Hofstede, \& Bond, 1991; Silverthorne, 2005). Uçanok (2009) explica cómo a través de los valores organizacionales los trabajadores interpretan sus experiencias en el contexto laboral, atribuyendo significados en términos de productividad, condiciones de trabajo y eventos que forman parte de este ámbito. Para Gelfand, Leslie, y Fehr (2008), en los tiempos actuales, los valores son los que van a definir el desarrollo de todas las ciencias, incluyendo los estudios transculturales.

Entre los valores definidos por la organización como uno de los componentes de la cultura organizacional, y los del trabajador, se va a plantear una relación que va a establecer el grado de congruencia entre ambos. Para Liedtka (1989) y Posner y Schmidt (1993), la congruencia de valores se puede manifestar como ajuste entre ellos, como desajuste y como conflicto entre valores personales y organizacionales. Este último tipo de relación se define por la existencia de valores organizacional opuestos a los del trabajador (Bouckenooghe, Buelens, Fontaine, \& Vanderheyden, 2005; Ramos et al., 2012).

De todas las formas de congruencia de valores mencionadas, va a ser el conflicto de valores el ele- mento que va a influir en la vivencia de estrés laboral (Bouckenooghe et al., 2005; Lamm, Gordon, \& Purser, 2010; Liedtka, 1989; Medina, Munduate, Dorado, Martínez, \& Guerra, 2005; Oliveira, Tenório, Souza, Pereira, \& Souza, 2002). Esto fue soportado por los estudios de Morais (2011) y Ramos y Jordão (2013a) en empresas portuguesas, que identificaron una correlación significativa y positiva entre el conflicto de valores y la vivencia de estrés laboral, argumentando la importancia del conflicto en comparación con el ajuste o el desajuste como otras formas de congruencia.

\section{El contexto organizacional definido por el sector y su relación con el estrés laboral}

El contexto de las organizaciones también está compuesto por elementos no culturales que, según Gelfand et al. (2008), operan como "amplificadores" de las dinámicas organizacionales. El sector de las organizaciones es uno de los elementos no culturales considerados en los estudios sobre estrés laboral, debido a las diferencias que se establecen en organizaciones públicas y privadas de acuerdo con la vivencia de estrés laboral, los estresores y las estrategias de coping al estrés (Malik, 2011; Ramos \& Jordão, 2013a; Sinha, 2012; Tyler, Carroll, \& Cunningham, 1991).

Hay autores que argumentan que esta diferencia por sector es debido a la estrecha relación entre el tipo de empresa y la cultura organizacional, que también será diferente en organizaciones públicas y privadas (Mazzola, Schonfeld, \& Spector, 2011; Ramos \& Jordão, 2012b, 2013a). De este modo, va a existir una relación entre el sector, la cultura definida por los valores y la vivencia de estrés laboral, incluyendo los estresores y las estrategias de coping dentro de este proceso.

\section{Relación entre el conflicto de valores, el sector de la organización y el proceso de estrés laboral}

La relación que se establece entre el conflicto, el proceso de estrés y el sector está determinada, entre otras, por el efecto del contexto como mediador perceptual de la vivencia de estrés, a partir de la forma como los estresores organizacionales van a ser identificados por parte de los trabajadores de una misma organización (Peterson \& Wilson, 2002). Por otra parte, la cultura de una organización va a permitir o no que sus tra- 
bajadores pongan en práctica determinadas estrategias de coping debido a la relación que se establece entre la cultura y el estrés laboral (Lansisalmi et al., 2000; Peiró et al., 2002). Por último, en este conjunto de relaciones vamos a incluir al sector de la organización, por la relación que establece con la cultura y los valores organizacionales, al existir un tipo de cultura específica en organizaciones públicas que las diferencias de las privadas. El sector también va a estar relacionado, entonces, con la vivencia de estrés, los estresores y las estrategias de coping, definiendo el comportamiento de estas variables que también se ha identificado como específico (Malik, 2011; Ramos \& Jordão, 2013b; Sinha, 2012; Tyler et al., 1991).

Por esta razón, nos propusimos como objetivo de investigación el desarrollo de un modelo que recoja las relaciones que se establecen entre la congruencia de valores considerando, específicamente, al conflicto entre valores organizacionales e individuales como forma de congruencia; el estrés laboral, los estresores y las estrategias de coping, que sea válido en función del sector de las organizaciones. Así, la información derivada de las relaciones descritas en el modelo podrán ser utilizadas por las organizaciones en función de minimizar el impacto negativo del estrés y generar estrategias más eficaces para lidiar con esta vivencia, humanizando los contextos de trabajo.
Un modelo descriptivo de relación entre el conflicto de valores, la vivencia de estrés laboral, las fuentes de estrés y las estrategias de coping, según el sector de la organización A partir del marco teórico descrito, representamos un modelo de relaciones (modelo inicial) en la figura 1.

En este modelo se describe la influencia que va a ejercer el sector tanto en el conflicto de valores como en el estrés laboral, las fuentes de estrés y las estrategias de coping. El conflicto de valores tendrá, entonces, un impacto directo en la vivencia de estrés y un efecto mediador de la influencia del sector en esta vivencia. Al mismo tiempo, el conflicto de valores, como elemento que caracteriza la cultura organizacional, también va a influir en el tipo de estresor identificado en las organizaciones y en las posibilidades de desarrollo de estrategias de coping al estrés por parte de los trabajadores. En este caso, establecimos que el conflicto de valores va a tener tanto un impacto directo en las estrategias de coping, como indirecto a través de la vivencia de estrés laboral y sus fuentes. Por último, identificamos que las estrategias de coping también van a derivar de las fuentes de estrés laboral, tanto de forma directa como indirecta a través de la vivencia estresante.

\section{Figura 1. Modelo teórico}

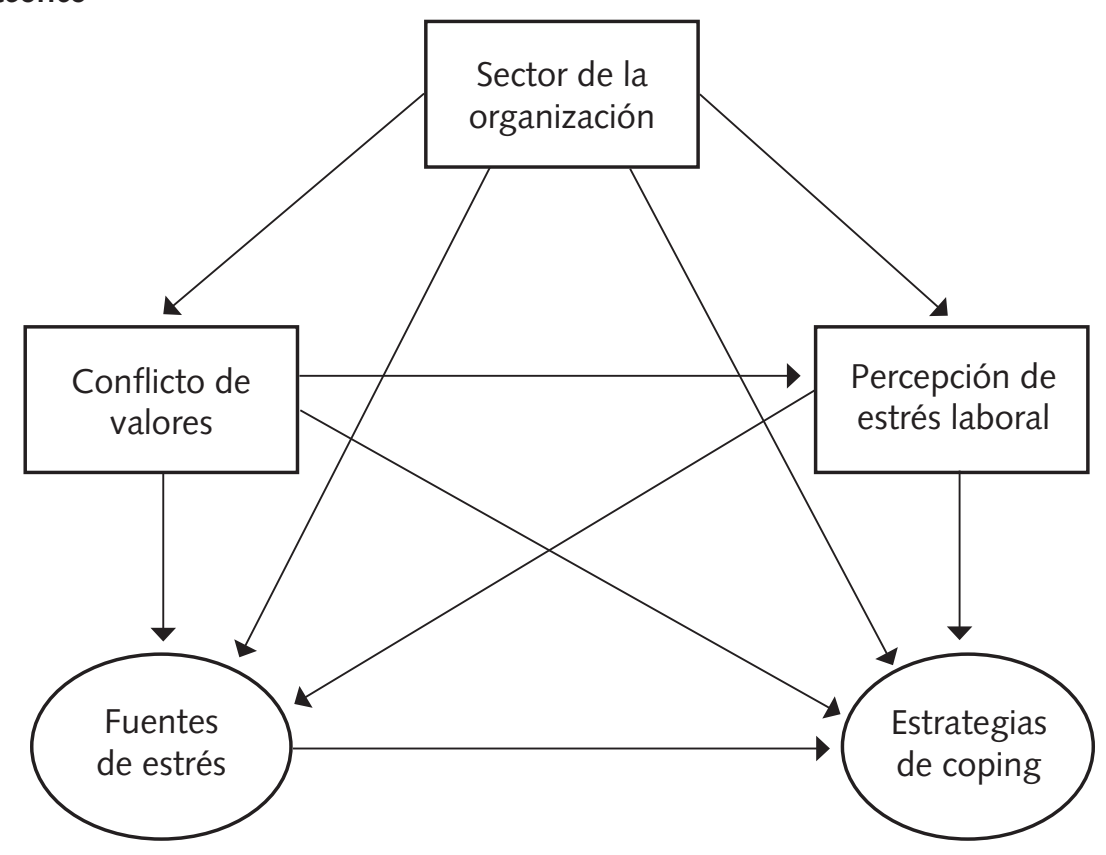




\section{MÉTODO}

\section{Muestra}

La muestra se constituyó por un total de 310 trabajadores distribuidos entre dos organizaciones de diferente sector $\left(\mathrm{N}_{\text {público }}=160 ; \mathrm{N}_{\text {privado }}=150\right)$. Ambas organizaciones son entidades del grupo de los servicios y se encuentran clasificadas como de gran tamaño de acuerdo con las normativas de la Comunidad Europea recogidas en el Código del Trabajo Portugués.

La muestra fue pareada de acuerdo con el género y la edad del trabajador (ver tabla 1). Para el pareamiento o matching se utilizó el paquete "Matching" del programa estadístico R (Team, 2011).

\section{Materiales y procedimiento}

Los datos en función de las variables identificadas en el modelo propuesto fueron recogidos a través de cuatro escalas validadas para la población portuguesa, donde se realizó el estudio.

\section{Materiales}

Los instrumentos, en función de las variables, son presentados a continuación.

Congruencia de valores: la congruencia de valores se determinó a partir de la aplicación del Inventario para la Articulación entre valores Personales y Organizacionales - APO de Ramos et al. (2012). Este inventario se distribuye en tres factores, explicando el $71.92 \%$ de la varianza, y mide el ajuste entre valores personales $(\alpha=.909)$, el conflicto entre valores personales y organizacionales $(\alpha=.891)$ y reconocimiento de valores organizacionales ( $\alpha=.901)$.

Fuentes de estrés laboral: fueron consideradas como fuentes de estrés laboral aquellas medidas por el Occupational Stress Inventory (OSI), validado para la población portuguesa por Cunha, Cooper, Moura, Reis, y Fernandes (1992). La escala de fuentes de presión que integra este instrumento, y que fue utilizada

Tabla 1. Distribución de la muestra por sector de las organizaciones

\begin{tabular}{|c|c|c|}
\hline & Público & Privado \\
\hline \multicolumn{3}{|l|}{ Género } \\
\hline Hombres & 64 & 60 \\
\hline Mujeres & 96 & 90 \\
\hline \multicolumn{3}{|l|}{ Estado civil } \\
\hline Solteros & 38 & 21 \\
\hline Casados & 90 & 101 \\
\hline Divorciados & 19 & 15 \\
\hline Otro & 13 & 13 \\
\hline \multicolumn{3}{|l|}{ Nivel de escolaridad } \\
\hline No graduados de nivel superior & 87 & 69 \\
\hline Graduados de nivel superior & 73 & 81 \\
\hline \multicolumn{3}{|l|}{ Edad (años) } \\
\hline Mínimo - máximo & $25-64$ & $28-65$ \\
\hline Media & 43.36 & 42.26 \\
\hline Desviación estándar & 9.138 & 8.183 \\
\hline \multicolumn{3}{|c|}{ Tiempo de trabajo en la organización (años) } \\
\hline Mínimo - máximo & $1-39$ & $1-3$ \\
\hline Media & 15.82 & 2.12 \\
\hline Desviación estándar & 9.501 & .679 \\
\hline
\end{tabular}

Nota: $\mathrm{N}_{\text {privado }}=150, \mathrm{~N}_{\text {público }}=160$ 
en el presente estudio, tuvo una consistencia interna elevada $(\alpha=.91)$. Esta escala está compuesta por 61 ítems, divididos en seis factores que a su vez son considerados como fuentes de estrés. Estas fuentes son los factores intrínsecos del puesto de trabajo $(\alpha=.65)$, el desempeño del rol $(\alpha=.66)$, las relaciones con los otros $(\alpha=.67)$, los logros conseguidos y el desarrollo de la carrera $(\alpha=.78)$, la estructura y el clima organizacional $(\alpha=.73)$ y la interface familia-trabajo $(\alpha=.66)$.

Percepción de estrés laboral: El estrés se midió de forma directa a partir de la percepción de los trabajadores por medio de la pregunta: “En qué grado se siente estresado debido a su trabajo?", para ser respondida de acuerdo con una escala de Likert de cinco puntos indicando: $0=$ Nulo, $1=$ Débil, $2=$ Moderado, $3=$ Fuerte y $4=$ Muy fuerte.

Estrategias de coping: Las estrategias de coping fueron identificadas a partir del cuestionario Brief Cope de Carver (1997), modificado para la población portuguesa por Ribeiro y Rodrigues (2004). Los factores identificados explican en esta versión el $67.7 \%$ de la varianza $\mathrm{y}$ fueron distribuidos en: coping activo $(\alpha=.65)$, planificación $(\alpha=.70)$, uso de apoyo instrumental $(\alpha=.81)$, uso de apoyo emocional $(\alpha=.79)$, religión $(\alpha=.80)$, reinterpretación positiva $(\alpha=.74)$, autoculpabilización $(\alpha=.62)$, aceptación $(\alpha=.55)$, expresión de sentimientos $(\alpha=.84)$, negación $(\alpha=.72)$, autodistracción $(\alpha=.67)$, falta de implicación $(\alpha=.78)$, uso de sustancias tóxicas $(\alpha=.81)$ y uso de humor $(\alpha=.83)$. Estos factores, a su vez, fueron reducidos a cuatro a través de un análisis factorial para facilitar su inclusión dentro del modelo estadístico $\left(\mathrm{R}^{2}=.5220 ; \alpha_{\text {control }}=.688 ; \alpha_{\text {relaciones }}=.664\right.$; $\left.\alpha_{\text {evitación }}=.397 ; \alpha_{\text {negación }}=.377\right)$.

Los datos sociodemográficos de los participantes fueron recogidos al final del inventario.

\section{Procedimiento}

El inventario compuesto por las cuatro escalas fue distribuido en ambas organizaciones de forma on-line a través del Google Docs (Google, 2010) y en la organización del sector público también se incorporó el formato en papel para los trabajadores sin acceso al correo electrónico o a la Internet. En ambos casos, los trabajadores tuvieron acceso al inventario durante un mes, y el equipo de investigadores contó para este fin con el apoyo de los departamentos de recursos huma- nos de ambas entidades, con el que se establecieron cronogramas de envío de materiales, la manera de recoger la información y retroalimentación del proceso.

\section{Análisis de la información}

Para identificar las relaciones entre variables se hicieron cálculos de correlaciones, tanto de forma general como para cada sector. La medida de significancia utilizada fue el coeficiente de correlación de Pearson. El modelo fue validado a partir del uso de ecuaciones estructurales. Los índices utilizados para verificar el ajuste fueron el índice comparativo del ajuste (CFI), el índice del ajuste de la bondad (GFI), el índice de bondad del ajuste de la parsimonia (PGFI), el índice de ajuste normado (NFI) y el error cuadrático medio de aproximación (RMSEA) de acuerdo con lo recomendando por la literatura (Arias, 2008; BatistaFoguet, Coenders, \& Alonso, 2004; De Bem, Lanzer, Tambosi Filho, Sanchez, \& Bernardi Junior, 2011).

Según estos parámetros el modelo es considerado aceptable si los índices CFI, GFI y NFI ultrapasan los 0.90 y excelente si ultrapasa los 0.95 , de acuerdo con autores como Arias (2008), Byrne (2010) y Marôco (2010). Estos mismos autores proponen valores de RMSEA entre 1.10 y 0.05 para que el modelo sea considerado como bueno, y valores inferiores a 0.05 para que el modelo sea considerado como excelente. Los valores del PGFI considerados como aceptables son aquellos que oscilan entre 0.6 y 0.8 , mientras que se considera como ideal tener valores superiores a 0.8 (Marôco, 2010).

El programa estadístico para la realización los cálculos de correlaciones fue el SPSS 20 (IBM, 2011). Para el modelo de ecuaciones estructurales se utilizó el paquete AMOS 19 (IBM, 2010).

\section{RESULTADOS Y DISCUSIÓN}

El modelo teórico propuesto fue diseñado siguiendo un sistema de ecuaciones estructurales y sometido a un cálculo de niveles de ajuste y validación de las correlaciones propuestas (ver figura 2).

A pesar de que el modelo inicial presentó buenos índices de ajuste $(\mathrm{CFI}=0.936, \mathrm{GFI}=0.915, \mathrm{NFI}=$ 0.911, RMSEA $=0.086$, PGFI $=0.583$, obtuvimos correlaciones no significativas entre las variables $\left(\beta_{\text {fuentes } \leqslant \text { sector }}=-.106 ; \beta_{\text {coping } \leqslant \text { conflicto }}=-.027 ; p>.005\right)$. 
De acuerdo con estos resultados, el sector de la organización no es determinante en la definición de las fuentes de estrés laboral de una forma directa. En este sentido podemos entonces definir que, a pesar de la existencia de estudios que argumentan que las organizaciones públicas y privadas van a tener fuentes de estrés específicas para cada una de ellas, nuestros resultados confirman resultados previos obtenidos para el contexto organizacional portugués, que indica no tener diferencias por sector en relación con los estresores en las organizaciones (Ramos \& Jordão, 2013b).

Los cálculos presentados señalan, además, que el conflicto de valores no ejerce una influencia directa de forma significativa en las estrategias de coping al estrés utilizadas. En el proceso de vivencia de estrés, el surgimiento de estrategias de coping puede ocurrir, en muchas ocasiones, sin que todavía aparezca el estrés laboral. Esto sucede cuando los trabajadores reconocen una situación como amenazadora y como generadora potencial de estrés (Schuler, 1985). De ahí que, por ejemplo, existan estrategias de coping que correla- cionen significativamente con las fuentes de estrés, sin que exista una correlación con la vivencia de estrés, de acuerdo con los resultados de Ramos y Jordão (2013b).

En el caso de la relación que se establece entre el conflicto de valores y las estrategias de coping, todo parece indicar que la situación de conflicto no es vista por el trabajador como una amenaza potencial de estrés, sino que, efectivamente, el surgimiento de un conflicto de valores va a derivar en estrés laboral. Por este motivo, la relación entre conflicto y coping siempre estaría mediada por la vivencia de estrés laboral. Esto podría argumentar el porqué de que esta correlación no sea significativa. Sin embargo, este resultado podría ser mejor explorado en futuros estudios que expliquen esta relación.

Los resultados de estas correlaciones nos llevaron a eliminar las relaciones indicadas entre sector y fuentes de estrés, y entre conflicto de valores y estrategias de coping. Así, elaboramos un nuevo modelo a partir de las relaciones significativas identificadas.

Figura 2. Resultados de las correlaciones entre las variables del modelo teórico a partir de un sistema de ecuaciones estructurales (modelo inicial).

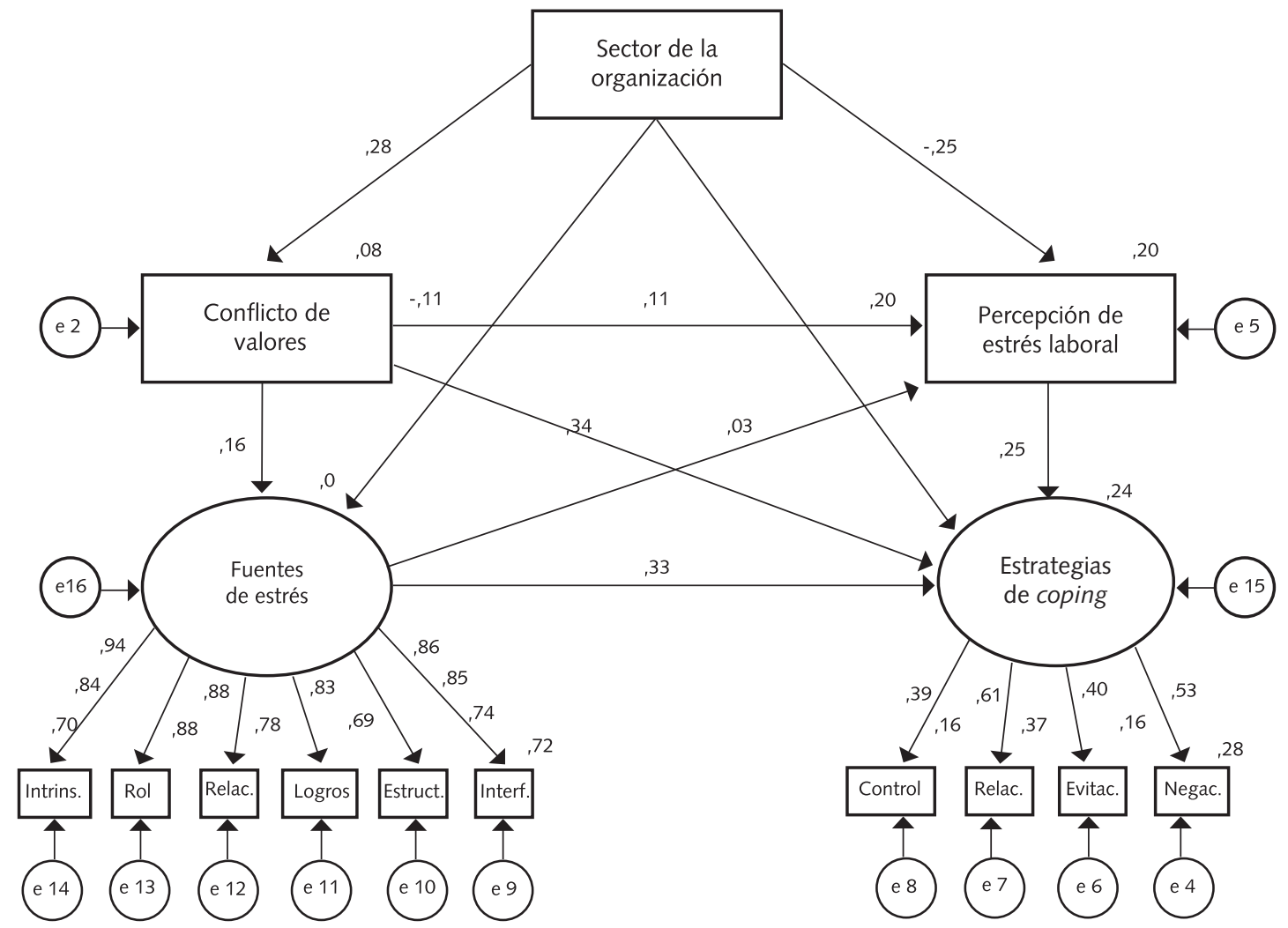


Resultados del rediseño del modelo inicial. Presentación del modelo final

El nuevo modelo fue diseñado manteniendo sólo las correlaciones significativas entre las variables encontradas previamente. El modelo rediseñado puede verse en la figura 3.

De acuerdo con los cálculos realizados, el modelo presenta todas las correlaciones entre las variables de forma significativa (ver tabla 2). Este modelo rediseñado presenta índices de ajuste que indican que puede ser considerado aceptable (CFI $=0.935$, GFI $=0.914$, $\mathrm{NFI}=0.909$, RMSEA $=0.085$, PGFI $=0.602$ ), por lo que queda definido como nuestro modelo final.

\section{CONCLUSIONES}

A partir de los cálculos estadísticos realizados, obtuvimos un modelo final que explica la relación que se establece entre el conflicto entre valores organizacionales y personales como elemento de la cultura y la con- gruencia de valores, las fuentes de estrés laboral, la percepción de estrés y las estrategias de coping al estrés (figura 3); donde resulta especialmente relevante el papel del contexto en el proceso de estrés laboral. Este modelo derivado de las correlaciones de las variables calculadas en el modelo inicial, es el más robusto en términos estadísticos comparado con los modelos anteriores.

Este modelo describe el efecto mediador del conflicto de valores de la relación entre el sector y el estrés laboral, lo que comprueba lo encontrado en nuestro marco teórico sobre el papel de la cultura organizacional en la vivencia de estrés. Al mismo tiempo, centra las relaciones de las variables asociadas al tipo de estrategias de coping en la vivencia de estrés laboral, que funciona como variable mediadora de esta relación. Por último, el modelo muestra que el conflicto de valores tiene un impacto indirecto en el desarrollo de estrategias de coping al estrés, mediante la vivencia de estrés laboral.

Figura 3. Resultados de las correlaciones entre las variables del modelo rediseñado a partir de un sistema de ecuaciones estructurales (modelo final).

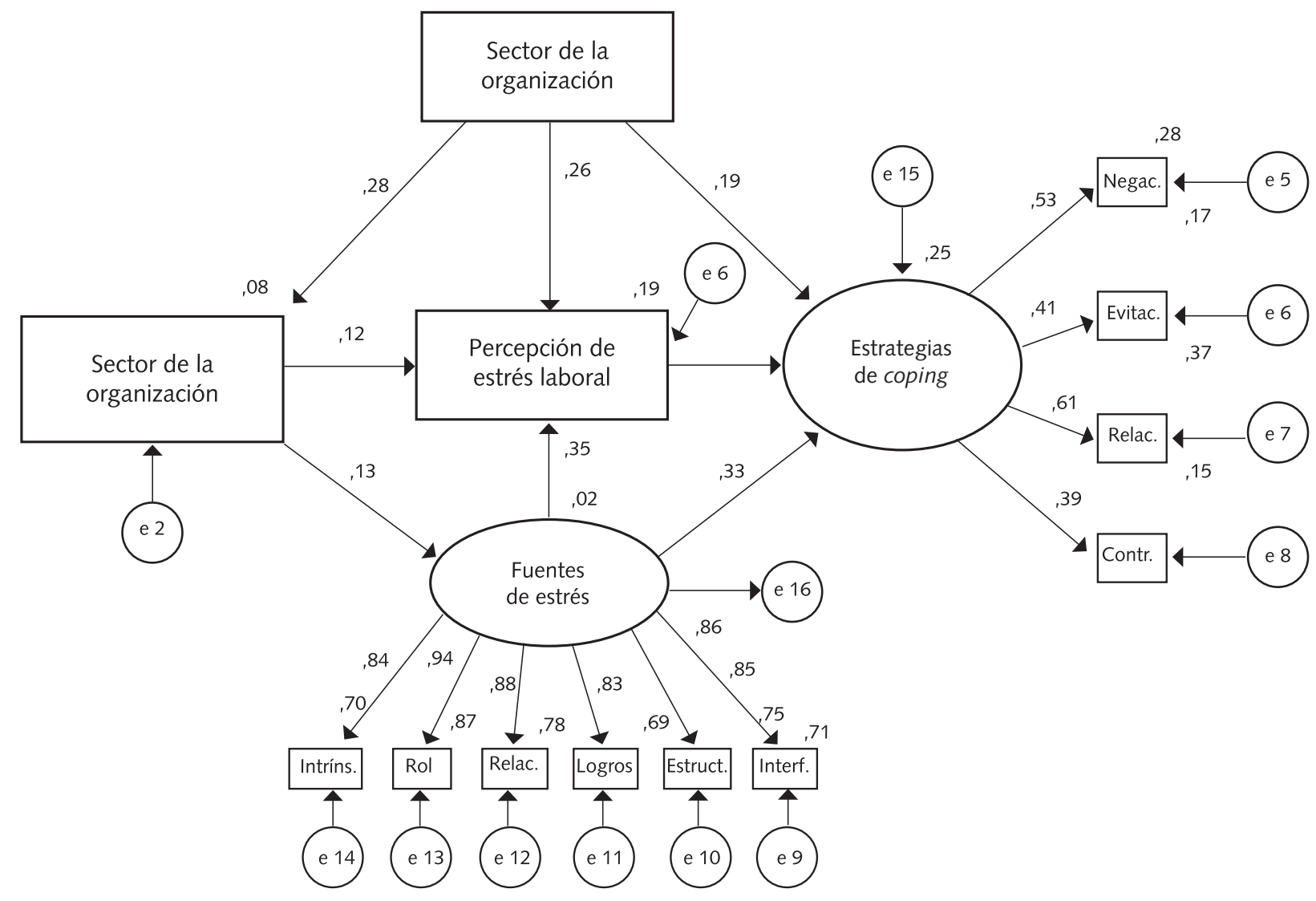


Tabla 2. Resultados del análisis del modelo de ecuaciones estructurales (modelo final)

\begin{tabular}{|c|c|c|c|c|c|}
\hline Modelo estructural & & & $\begin{array}{l}\text { Estimados } \\
\text { estandarizados }\end{array}$ & $\begin{array}{c}\text { Error } \\
\text { estándar }\end{array}$ & $\begin{array}{l}\text { Ratio Crítico } \\
\text { (CR) }\end{array}$ \\
\hline Conflicto de valores & $\leftarrow$ & Sector de la organización & .283 &, 864 & $5,188^{* *}$ \\
\hline Percepción de estrés laboral & $\leftarrow$ & Fuentes de estrés & .346 &, 005 & $6,384 * *$ \\
\hline Percepción de estrés laboral & $\leftarrow$ & Sector de la organización & -.257 & ,095 & $-4,785^{* *}$ \\
\hline Percepción de estrés laboral & $\leftarrow$ & Conflicto de Valores & .117 &, 006 & $2,183^{*}$ \\
\hline Estrategias de coping & $\leftarrow$ & Fuentes de estrés & .328 & ,017 & $3,795 * *$ \\
\hline Estrategias de coping & $\leftarrow$ & Percepción de estrés laboral & .247 & 189 & $3,012^{*}$ \\
\hline Estrategias de coping & $\leftarrow$ & Sector de la organización & .193 & ,305 & $2,568^{*}$ \\
\hline Coping de negacióna & $\leftarrow$ & Estrategias de coping & .525 & & \\
\hline Coping de evitación & $\leftarrow$ & Estrategias de coping & .409 & 097 & $4,627^{* *}$ \\
\hline Coping de relaciones & $\leftarrow$ & Estrategias de coping & .611 & ,203 & $5,431 * *$ \\
\hline Coping de control & $\leftarrow$ & Estrategias de coping & .387 &, 167 & $4,465^{* *}$ \\
\hline $\begin{array}{l}\text { Fuentes vinculadas a la } \\
\text { interface trabajo/casa }\end{array}$ & $\leftarrow$ & Fuentes de estrés & .845 & & \\
\hline $\begin{array}{l}\text { Fuentes vinculadas a la } \\
\text { estructura }\end{array}$ & $\leftarrow$ & Fuentes de estrés & .863 &, 044 & $19,787^{* *}$ \\
\hline Fuentes vinculadas a logros & $\leftarrow$ & Fuentes de estrés & .834 & ,041 & $18,638 * *$ \\
\hline $\begin{array}{l}\text { Fuentes vinculadas } \\
\text { a relaciones }\end{array}$ & $\leftarrow$ & Fuentes de estrés & .883 & ,040 & $20,602 * *$ \\
\hline $\begin{array}{l}\text { Fuentes relacionadas } \\
\text { a los roles }\end{array}$ & $\leftarrow$ & Fuentes de estrés & .935 & ,043 & $22,931 * *$ \\
\hline Fuentes intrínsecas & $\leftarrow$ & Fuentes de estrés & .838 & ,036 & $18,792 * *$ \\
\hline
\end{tabular}

Nota: ${ }^{a}$ indica que el parámetro fue fijado. ${ }^{*}$ indica $p<.05 ;{ }^{* *}$ indica $p<.01$

Estas relaciones reflejan, en primer lugar, la importancia del contexto definido por elementos culturales y no culturales en la vivencia de estrés laboral, incluyendo las fuentes de estrés y las estrategias de coping, elemento explorado por Ramos y Jordão (2013a). En este sentido, la vivencia de estrés laboral debería dejar de ser considerada como un fenómeno independiente del contexto organizacional, tal y como ha sido estudiado generalmente, para pasar a ser un fenómenos derivado de la influencia de las características culturales y no culturales de la organización donde se manifiesta este proceso. Nuestro estudio, en segundo lugar, también muestra al conflicto de valores como el elemento de la congruencia de valores que resulta significativo para el estudio del estrés en las organizaciones, confirmando resultados anteriores obtenidos por Ramos y Jordão (2012a) y Ramos y Jordão (2013a). Por último, ofrece una perspectiva en las relaciones entre las variables estudiadas que no ha sido considerada anteriormente en los estudios sobre cultura y estrés laboral, a partir de las relaciones significativas presentadas y los efectos mediadores de las variables encontradas. 


\section{REFERENCIAS}

Arias, B. (2008). Desarrollo de un ejemplo de análisis factorial confirmatorio con lisrel, amos y sas. Paper presented at the Seminario de Actualización en Investigación sobre Discapacidad, Valladolid.

Batista-Foguet, J.M., Coenders, G., \& Alonso, J. (2004). [confirmatory factor analysis. Its role on the validation of health related questionnaires]. Medicina Clínica, 122 Suppl 1, 21-27.

Bouckenooghe, D., Buelens, M., Fontaine, J., \& Vanderheyden, K. (2005). The prediction of stress by values and value conflict. The Journal of Psychology, 139(4), 369-382.

Byrne, B.M. (2010). Structural equation modeling with amos: Basic concepts, applications, and programming (2nd ed.). New York, NY US: Routledge/Taylor \& Francis Group.

Carver, C. (1997). You want to measure coping but your protocol's too long: Consider the brief cope. International Journal of Behavioral Medicine, 4(1), 92-100.

Cunha, R.C., Cooper, C.L., Moura, M.I., Reis, M.E., \& Fernandes, P. (1992). Portuguese version of the osi: A study of reliability and validity. Stress Medicine, 8(4), 247-251.

De Bem, A.B., Lanzer, E.A., Tambosi Filho, E., Sanchez, O.P., \& Bernardi Junior, P. (2011). Validade e confiabilidade de instrumento de avaliação da docência sob a ótica dos modelos de equação estrutural. Avaliação: Revista da Avaliação da Educação Superior (Campinas), 16, 375-401.

Dolan, S., \& García, S. (2002). Managing by values. Cultural redesign for strategic organizational change at the dawn of the twenty-first century. Journal of Management Development, 21 (2), 101.

Erkutlu, H., Chafra, J., \& Bumin, B. (2011). Organizational culture's role in the relationship between power bases and job stress. Hacettepe University Journal of Education, 40, 198-209.

Franke, R.H., Hofstede, G., \& Bond, M.H. (1991). Cultural roots of economic performance: A research note. Strategic Management Journal, 12, 165-173.

Ganster, D.C., \& Rosen, C.C. (2013). Work stress and employee health: A multidisciplinary review. Journal of Management, 39(5), 1085-1122. doi: $10.1177 / 0149206313475815$
Gelfand, M.J., Leslie, L.M., \& Fehr, R. (2008). To prosper, organizational psychology should... adopt a global perspective. Journal of Organizational Behavior, 29(4), 493-517. doi: 10.1002/job.530

Goh, Y.W., Sawang, S., \& Oei, T.P.S. (2010). The revised transactional model (rtm) of occupational stress and coping: An improved process approach. Australian \& New Zealand Journal of Organisational Psychology, 3(1), 13-20. doi: 10.1375/ajop.3.1.13

Google. (2010). Google docs. California. Retrieved from https://www.google.com/

Hofstede, G., Neuijen, B., Ohayv, D.D., \& Sanders, G. (1990). Measuring organizational cultures: A qualitative and quantitative study across twenty cases. Administrative Science Quarterly, 35, 286-316.

IBM. (2010). Ibm-spss-amos. New York: IBM.

IBM. (2011). Ibm spss statistic (Version 20). New York: IBM.

Lamm, E., Gordon, J.R., \& Purser, R.E. (2010). The role of value congruence in organizational change. Organization Development Journal, 28(2), 49-64.

Lansisalmi, H., Peiró, J., \& Kivimaki, M. (2000). Collective stress and coping in the context of organizational culture. European Journal of Work \& Organizational Psychology, 9(4), 527-559. doi: 10.1080/13594320050203120

Latack, J.C., \& Havlovic, S.J. (1992). Coping with job stress: A conceptual evaluation framework for coping measures. Journal of Organizational Behavior, 13(5), 479-508. doi: 10.1002/job.4030130505

Liedtka, J.M. (1989). Value congruence: The interplay of individual and organizational value systems. Journal of Business Ethics, 8(10), 805-815.

Macklin, D.S., Smith, L.A., \& Dollard, M.F. (2006). Public and private sector work stress: Workers compensation, levels of distress and job satisfaction, and the demand-control-support model. Australian Journal of Psychology, 58(3), 130-143. doi: 10.1080/00049530600940190

Malik, N. (2011). A study on occupational stress experienced by private and public banks employees in quetta city. African Journal of Business Management, 5(8), 3063-3070. doi: 10.5897/AJBM10.199

Mark, G.M., \& Smith, A.P. (2008). Stress models: A review and suggested new direction In J. Houdmont 
\& S. Leka (Eds.), Occupational health psychology, european perspectives on research, education and practice (Vol. 3, pp. 111-144). Nottingham: Nottingham University Press.

Marôco, J. (2010). Análise de equações estruturais. Fundamentos teóricos, software \& aplicações. Porto: ReportNumber.

Mazzola, J.J., Schonfeld, I.S., \& Spector, P.E. (2011). What qualitative research has taught us about occupational stress. Stress \& Health: Journal of the International Society for the Investigation of Stress, 27(2), 93-110.

Medina, F.J., Munduate, L., Dorado, M.A., Martínez, I., \& Guerra, J.M. (2005). Types of intragroup conflict and affective reactions. Journal of Managerial Psychology, 20(3-4), 219-230. doi: 10.1108/02683940510589019

Monat, A., \& Lazarus, R.S. (1991). Stress and coping : An anthology (3 ed.). New York: Columbia University Press.

Morais, T. (2011). Cultura organizacional e stress ocupacional - a articulação entre valores individuais e organizacionais e a sua relação com a perceção de stress ocupacional. (Mestrado integrado em Psicologia), Universidade do Porto, Porto.

Morais, T., Ramos, V., \& Jordão, F. (2012). A articulação entre valores individuais e organizacionais e a sua relação com a perceção de stress ocupacional. Paper presented at the $1^{\circ}$ Congresso Nacional da Ordem dos Psicólogos Portugueses, Lisbon.

Nazari, K., \& Emami, M. (2012). The investigation of the relation between job stress and job satisfaction (case study in faculty members of recognized public and private universities in the province of kermanshah). Advances in Natural \& Applied Sciences, 6(2), 219-229.

Oliveira, L., Tenório, J.C., Souza, A.L.d., Pereira, E.A., \& Souza, W. (2002). A síndrome de burnout e os valores organizacionais: Um estudo comparativo em hospitais universitários. Revista Psicología: reflexión y crítica, 15(1), 189 - 200.

Peiró, J., Zurriaga, R., \& González-Romá, V. (2002). Análisis y diagnóstico de las situaciones y experiencias de estrés colectivo en las unidades de trabajo y en las organizaciones de servicios sociales. Prevención, trabajo y salud: Revista del Instituto Nacional de Seguridad e Higiene en el Trabajo, 20, 11-21.
Peterson, M., \& Wilson, J.F. (2002). The culture-workhealth model and work stress. American Journal of Health Behavior, 26(1), 16-24.

Posner, B.Z., \& Schmidt, W.H. (1993). Values congruence and differences between the interplay of personal and organizational value systems. Journal of Business Ethics, 12(5), 341-347.

Ramos, V., \& Jordão, F. (2012a). Género y estrés laboral: Estudio de semejanzas y diferencias de acuerdo con factores de riesgo y mecanismos de coping. Revista Psicologia: Organizações e Trabalho(s/n).

Ramos, V., \& Jordão, F. (2012b). The relationship between cultural and non-cultural elements as a way to describe organizational context. Psicologia.com, 16(7). http://hdl.handle.net/10401/5459

Ramos, V., \& Jordão, F. (2013a). Are public workers more stressed than private workers? The relationship between work stress and value congruence. Psychology Research, 3(7).

Ramos, V., \& Jordão, F. (2013b). La relación entre el estrés laboral, las fuentes que le dan origen y las estrategias de coping en el sector público y el privado. FPCE-UP.

Ramos, V., Jordão, F., \& Morais, T. (2012). Desarrollo y validación del inventario para medir la articulación entre la persona y la organización (inventario apo) Evaluar, 12, 60 - 82 .

Lei n. ${ }^{\circ} 7 / 2009$ de 12 de fevereiro que aprova a revisão do código do trabalho, 1 C.F.R. (2009).

Ribeiro, J.L.P., \& Rodrigues, A.P. (2004). Questões acerca do coping: A propósito do estudo de adaptação do brief cope. Psicologia, Saúde \& Doenças, 5 (1), 3-15.

Schein, E.H. (1996). Culture: The missing concept in organization studies. Administrative Science Quarterly, 41(2), 229-240.

Schuler, R.S. (1985). An integrative transactional process model of coping with stress in organizations. In T. A. Beehr \& R. S. Bhagat (Eds.), Human stress and cognition in organizations: An integrated perspective (pp. 347-374). New York: Wiley.

Schwartz, S.H. (1992). Universals in the content and structure of values: Theoretical advances and empirical tests in 20 countries. In M. Zanna (Ed.), Advances in experimental social psychology (Vol. 25, pp. 1-65). New York: Academic Press. 
Silverthorne, C.P. (2005). Organizational psychology in cross-cultural perspective. New York: New York University Press.

Singh, A.P., \& Singh, S. (2009). Effects of stress and work culture on job satisfaction. ICFAI Journal of Organizational Behavior, 8(2), 52-62.

Sinha, R. (2012). Stress management--a comparative study of selected public and private sector organization in chhattisgarh. International Journal of Research in Commerce and Management, 3(3), 126-130. doi: http://ijrcm.org.in/commerce/index.php

Srivastav, A.K. (2009). Control climate in public sector: Relationship with role stress, coping strategy and personal variables. Pranjana: The Journal of Management Awareness, 12(1), 51-63.
Team, R.D.C. (2011). R: A language and environment for statistical computing. Vienna: R Foundation for Statistical Computing. Recuperado de http://www.Rproject.org/.

Tyler, P.A., Carroll, D., \& Cunningham, S.E. (1991). Stress and well-being in nurses: A comparison of the public and private sectors. International Journal of Nursing Studies, 28(2), 125-130. doi: 10.1016/00207489(91)90002-k

Uçanok, B. (2009). The effects of work values, work-value congruence and work centrality on organizational citizenship behavior. International Journal of Human and Social Sciences 4(9), 626-639. 